


\title{
EL EMPRENDIMIENTO SOCIAL: UN TERRITORIO DE APRENDIZAJE PARA LA SUPERACIÓN DE UN MODELO EN CRISIS
}

\author{
Autor: Amparo Merino de Diego. ${ }^{1}$ \\ Departamento de Gestión Empresarial. \\ Facultad de Ciencias Económicas y Empresariales. \\ Universidad Pontificia Comillas.
}

\section{Resumen}

Este trabajo toma como punto de partida la crisis ecosocial que acompaña a la dinámica expansiva del capitalismo y se pregunta si hay algún espacio en la arena empresarial para hacer frente a este desafío global. Se utiliza un estilo argumentativo para defender que la empresa social representa una respuesta eficaz por tres razones: 1) ayuda a ampliar la consciencia sobre las contradicciones del modelo económico capitalista; 2) constituye un ejemplo de innovación en el área de las transiciones a la sostenibilidad; y 3) ofrece un espacio favorable para avanzar hacia un gobierno de la empresa más democrático y una responsabilidad empresarial más auténtica.

\footnotetext{
${ }^{1}$ amerino@cee.upcomillas.es
} 
Palabras clave: emprendimiento social; empresa social; crisis ecosocial; capitalismo; transiciones a la sostenibilidad.

Social entrepreneurship: A learning arena to transcend a model in crisis.

\begin{abstract}
This paper has as a starting point the ecological and social crisis linked to the expansive dynamics of capitalism. We wonder if there is some room in the business management arena to deal with this global challenge. An argumentative style is used to defend that social businesses are an effective response for three reasons: 1 ) it helps to deepen the awareness of the contradictions of capitalism and its model of thinking; 2 ) it illustrates a model of innovation in the area of transitions to sustainability; and 3 ) it is a practical experience to advance a more democratic business governance and more genuinely business responsibility.
\end{abstract}

Key words: social entrepreneurship; social business; ecological and social crisis; capitalism; transitions to sustainability.

\title{
1. INTRODUCCIÓN.
}

La actual crisis económico-financiera puede ser interpretada como una más de las crisis consustanciales a nuestro modelo de producción y consumo dominante. Como argumenta Harvey (2010), la expansión exponencial del capital y la necesaria continuidad de su flujo creciente marcan el funcionamiento del capitalismo y su historia. Una historia que es más bien la de la búsqueda de distintas formas de sortear los límites al crecimiento y circulación del capital.

El imperativo de una acumulación perpetua, acompañado de la capacidad técnica que supuso la Revolución industrial y del contexto ideológico en el que se gestó ${ }^{2}$, se ha traducido en una espiral de crecimiento indefinido que lleva consigo un límite que se está mostrando cada vez más difícil de soslayar, especialmente desde la segunda mitad del siglo XX: las presiones ejercidas por la actividad hu-

\footnotetext{
${ }^{2}$ Contexto marcado por el concepto de liberalismo y de propiedad privada, por la consideración del mercado como marco privilegiado para la satisfacción de necesidades y por la búsqueda de la máxima utilidad individual.
} 
mana sobre la biosfera han alcanzado una escala tal que implican la posibilidad de cambios ambientales abruptos o irreversibles, con las imprevisibles consecuencias sobre la supervivencia y bienestar de amplios sectores de la población mundial.

Efectivamente, Rockström et al. (2009) han identificado y cuantificado nueve líneas rojas cuya trasgresión entraña el riesgo de tales cambios irreversibles, ya sea a escala continental o planetaria: emisiones descontroladas de $\mathrm{CO}^{2}$, acidificación de los océanos, reducción de la capa de ozono estratosférico, alteración de los ciclos biogeoquímicos del nitrógeno y del fósforo, uso excesivo de agua dulce, cambios en los usos del suelo, aceleración de la pérdida de biodiversidad, dispersión de químicos y aumento de la carga de aerosoles en la atmósfera. A pesar de la complejidad de determinar el límite preciso de estas fronteras, los análisis desarrollados por este reconocido grupo de investigadores ${ }^{3}$ señalan que varias de estas líneas rojas ya se han traspasado, con la consiguiente incertidumbre acerca del horizonte temporal en el que tal traslimitación no generará drásticos cambios ambientales globales.

Por otro lado, los análisis sobre la huella global ejercida por la actividad humana sobre la biosfera ponen de manifiesto cómo se está superando su capacidad para regenerar los recursos demandados y absorber los residuos generados. Según los cálculos de la Global Footprint Network, la humanidad demandó los recursos y servicios de 1,51 planetas en 2007, cifra que se ha multiplicado por 2,5 veces desde 1961 (Ewing et al., 2010). En la misma línea, la Evaluación de los Ecosistemas del Milenio, auspiciada por la UNEP (Millennium EcosystemAssessment, 2005), concluye que los cambios realizados en los ecosistemas, si bien han contribuido a generar considerables ganancias netas en el bienestar de muchos seres humanos, esto ha sucedido con crecientes costes consistentes en la degradación los ecosistemas, un mayor riesgo de cambios no lineales y la acentuación de la pobreza de importantes grupos de personas ${ }^{4}$.

El reconocimiento de estos límites, de hecho, no tiene nada de novedoso. Ya hace más de siglo y medio Mill (1848: 311) verbalizaba en sus Principios de Economía Política el inevitable camino hacia el estado estacionario de las economías: "It must always have been seen, more or less distinctively, by political economists, that the increase of wealth is not boundless; that at the end of what they term the progressive state lies the stationary state, that all progress in wealth is but a

${ }^{3}$ Pertenecientes al Stockolm Resilience Centre (Universidad de Estocolmo), organismo dedicado al estudio sobre el gobierno de sistemas socio-ecológicos con un especial énfasis en su resiliencia. Véase http://www.stockholmresilience.org/21/research/research-programmes/planetary-boundaries.html (acceso: $4 / 12 / 2013)$.

4 Véase en este sentido la argumentación desarrollada por NEF (2006) para cuestionar la idea del crecimiento económico como condición previa para la reducción de la pobreza, y para mostrar su menor eficacia frente a políticas distributivas, aparte de los costes ecológicos que, como hemos visto, lleva aparejados. 
postponement of this, and that each step in advance is an approach to it". Ciertamente, desde el siglo XVIII la disciplina económica había venido progresivamente marginando de su centro de atención la idea fisiocrática de extracción de riqueza generada por la Tierra, para sustituirla por una noción de riqueza producida por el ser humano y su deseable crecimiento. Así, el razonamiento económico fue dominando el lenguaje de la economía hasta desconectarla de los conflictos sociales y/o ambientales inherentes al proceso económico (Naredo, 2003).

Este contexto de supremacía del razonamiento económico maximizador supuso el caldo de cultivo necesario para que una noción de empresa guiada por idénticas bases fuera adquiriendo un protagonismo y predominio crecientes: el proceso de cambio económico e institucional que supuso el paso de una economía feudal a otra capitalista incrementó espectacularmente las oportunidades de negocio fruto del crecimiento de los mercados, del cambio tecnológico de la revolución industrial, de la creación de una abundante fuerza de trabajo asalariada y de un marco de incentivos favorable a la iniciativa individual y la propiedad privada. Cambios que otorgaron un intenso protagonismo al "papel del empresario como coordinador del proceso productivo y de la empresa privada como modelo organizativo, a costa por ejemplo de sistemas productivos cooperativos o comunales" (Valdaliso y López, 2000: 178). Y los fenómenos globalizadores de las últimas décadas han facilitado el aumento de poder de grandes corporaciones transnacionales que gobiernan y controlan extensas redes de valor global. Este predominio ha acabado condicionando y construyendo las dimensiones económica, política y cultural de nuestras vidas y, en definitiva, lo que entendemos por bienestar personal y colectivo (Bendell, 2004) .

Nos encontramos, pues, ante un escenario marcado 1) por los conflictos entre el modelo capitalista expansivo y las realidades sociales y físicas más amplias; y 2) por la acción protagonista en ese modelo de unos agentes empresariales que operan sobre la base del crecimiento y la competencia coercitivos (Harvey, 2010), ejerciendo un amplio poder sobre las esferas individual y social de la vida. En tal escenario, cabe preguntarse si hay algún espacio para abordar las tensiones de la crisis de sostenibilidad desde la arena empresarial. Avanzar una respuesta a tal pregunta constituye el objetivo general de este trabajo: ¿es posible, y bajo qué condiciones, que la actividad empresarial y los principios que guían su gestión sean compatibles con un significado de la sostenibilidad no banalizado, ligado al mantenimiento de los ciclos de la Naturaleza, al cuidado como valor esencial y al florecimiento humano como fin último?

\footnotetext{
${ }^{5}$ De modo paralelo a ese poder en aumento, los procesos de regulación de la actividad empresarial se han ido haciendo más complejos, con la ortodoxia neoliberal en los años 70, el predominio de la responsabilidad social corporativa como práctica voluntaria en los 90 y, de modo más reciente, nuevas formas de regulación asumidas por $\mathrm{ONG}$, instituciones multistakeholder y alianzas público-privadas crecientemente apoyadas por organizaciones gubernamentales e intergubernamentales (Utting, 2005).
} 
Para abordar la cuestión, este texto centra la atención en un tipo de actor empresarial específico: aquél que tiene su razón de ser y su guía de acción no en un fin económico, sino en un fin más amplio, ligado a las condiciones que hacen sostenible a un sistema económico ${ }^{6}$. Un tipo de empresa que nace desde la consciencia de un problema sistémico y de la voluntad de cambiarlo. Esta idea de organización puede recibir diversas denominaciones, entre las que se incluye la de empresa ética o empresa sostenible, si bien se ha hecho más común la de empresa social (social business) o emprendimiento social (social entrepreneurship).

Pero, independientemente de su nombre y su significado preciso (cuestión que será abordada más adelante), lo cierto es que el fenómeno está adquiriendo una atención e interés crecientes por su potencial contribución a una vida buena y sostenible a través de sus distintos formatos y áreas de actuación. Interés que contrasta con el relativamente escaso desarrollo de la investigación sobre emprendimiento social, mucho menor que sobre el emprendimiento económico (Swanson y Zhang, 2010; Short et al., 2009; CASE, 2008; Light, 2008). En parte, la disparidad sobre la acotación de su significado y sobre las formas que puede adoptar debilita a la empresa social como campo de estudio. Pero, prioritariamente, su desarrollo como área de investigación requiere, junto a unas condiciones políticas, sociales y económicas favorables, la construcción de una comunidad de conocimiento y de práctica del emprendimiento social que sea activa y no fragmentada (CASE, 2008).

De ahí que este trabajo pretenda generar una contribución más al avance de esta área de conocimiento a través de su objetivo específico: defender que las empresas sociales constituyen una experiencia en la arena empresarial que puede resultar una vía de cambio eficaz (una más) ante la crisis de insostenibilidad global que acompaña al sistema económico vigente.

Para ello, tras delimitar el significado de empresa social que usamos en el desarrollo del trabajo, el texto se estructura en torno a tres líneas argumentales orientadas a sustentar la afirmación anterior. La primera defiende la utilidad del emprendimiento social como un ejercicio de cuestionamiento de las asunciones básicas que cimentan el modelo capitalista. La segunda se centra en el papel de las empresas sociales como un espacio de aprendizaje (desde enfoques pragmáticos) dentro del nuevo campo de conocimiento de las transiciones a la sostenibilidad. El tercer argumento sostiene que la empresa social es un formato propicio para avanzar hacia un gobierno empresarial construido sobre bases más democráticas y un ejercicio de responsabilidad empresarial más auténtico. El trabajo concluye extrayendo los ele-

\footnotetext{
${ }^{6}$ Condiciones que, de acuerdo con Recio (2010), son: 1) ecológicas, de modo que el empleo de recursos para generar bienestar se limita de forma permanente a un tamaño y una calidad que no sobreexplote las fuentes ni sobrecargue los sumideros que proporciona la ecosfera; 2) sociales, relacionadas con el acceso a bienes y servicios adecuados a la cobertura de necesidades básicas y la carga de trabajo (mercantil y no mercantil) que afecta a cada individuo; y 3 ) económicas, en el sentido de que mantenga un equilibrio entre producción, distribución y utilización del producto social.
} 
mentos esenciales de la argumentación previa y planteando caminos que se abren en el desarrollo del emprendimiento social como área de conocimiento.

\section{LA EMPRESA GUÍADA POR UNA MISIÓN SOCIAL.}

No hay en la literatura sobre emprendimiento social una definición monolítica del término que introdujera en los años 80 Bill Drayton, fundador de Ashoka, para ayudar a las organizaciones no lucrativas a explorar nuevas fuentes de ingresos (Dees, 2007). Tampoco hay una clasificación cerrada del tipo de organizaciones que se pueden incluir dentro de esta noción e, incluso, pone de manifiesto cómo se difuminan las fronteras entre los tradicionalmente distantes sector empresarial y sector no lucrativo (CASE, 2008; Dees, 2001). Sin embargo, es habitual encontrar en la idea de emprendimiento social la referencia a tres elementos centrales:

1. La misión social como su motor, frente al ánimo de lucro, condicionando la percepción y la valoración de lo que es una oportunidad de negocio (Comisión Europea, 2013; Yunus, 2011; NEF, 2005; Dees, 2001).

2. El impacto que genera identificando y resolviendo problemas sociales como pobreza, exclusión laboral, enfermedad, violación de derechos humanos o corrupción, entre otros (Ashoka, 2012; Bornstein y Davis, 2010; Dees, 2007; Drayton, 2005; Ducci et al., 2002).

3. La manera innovadora de organizar los recursos para ofrecer soluciones prácticas (Comisión Europea, 2013; Drayton, 2005).

A pesar de los diferentes énfasis que puede recibir la definición de emprendimiento social, existe consenso en torno a su objetivo de buscar un cambio sistémico, un nuevo equilibrio social (Ashoka, 2012; Swanson y Zhang, 2010; Light, 2008). En definitiva, es crucial el elemento del que arrancaría cualquier emprendimiento social: la consciencia sobre la existencia de un problema social y la voluntad de cambiarlo. Como ya adelantamos, entendemos aquí "problema social" como los desequilibrios que dificultan el bienestar, el cuidado, el florecimiento humano y la capacidad de los ritmos de la Tierra para sostenerlos.

Este punto de partida está presente en organizaciones no empresariales, como es el caso de la provisión de servicios sociales por organismos públicos o por entidades sin ánimo de lucro. Asimismo, las empresas con misión económica pueden generar soluciones a problemas sociales a través de su actividad filantrópica o guiadas por la lógica de la responsabilidad social corporativa (en adelante, RSC). En este sentido, Yunus (2008) argumenta a favor de la empresa social por contraste con las limitaciones del papel de gobiernos, instituciones multilaterales, ONG o empresas convencionales para luchar contra la pobreza. Defiende la fuerza del mercado para abordar problemas 
de pobreza global y degradación ambiental pero no "si sirve única e implacablemente a los objetivos económicos de sus accionistas más ricos" (Yunus, 2008: 25). También la Comisión Europea (2013: 31) explicita que lo que distingue a la empresa social de las organizaciones caritativas tradicionales es el hecho de que las primeras obtienen una proporción sustancial de sus ingresos a través de una actividad comercial, superior a la que pueden recibir a través de subvenciones o donaciones; y lo que la diferencia de las empresas convencionales es su misión primordialmente social.

En cualquier caso, aunque no hay consenso en torno a qué tipo de agentes cabría encuadrar dentro de los emprendedores sociales (Dees, 2001), este trabajo se alinea con el mencionado aprovechamiento de la fuerza del mercado (aunque no sólo) como parte de la definición de empresa social: desde la consciencia acerca de un problema social como motor del deseo de encontrar soluciones, partiría la creación de valor social como fin último y razón de ser de esta organización, pero usando para ello (aunque no sólo) estrategias de mercado y herramientas y conceptos típicamente probados por empresas convencionales (Merino, 2012).

Swanson y Zhang (2010) ofrecen un marco de gran utilidad para representar la gama de posibilidades que surgen para lograr el cambio social a través de prácticas empresariales, más allá de formas jurídicas concretas, tal y como se recoge en la Figura 1.

\section{Figura 1. Zona de emprendimiento social}

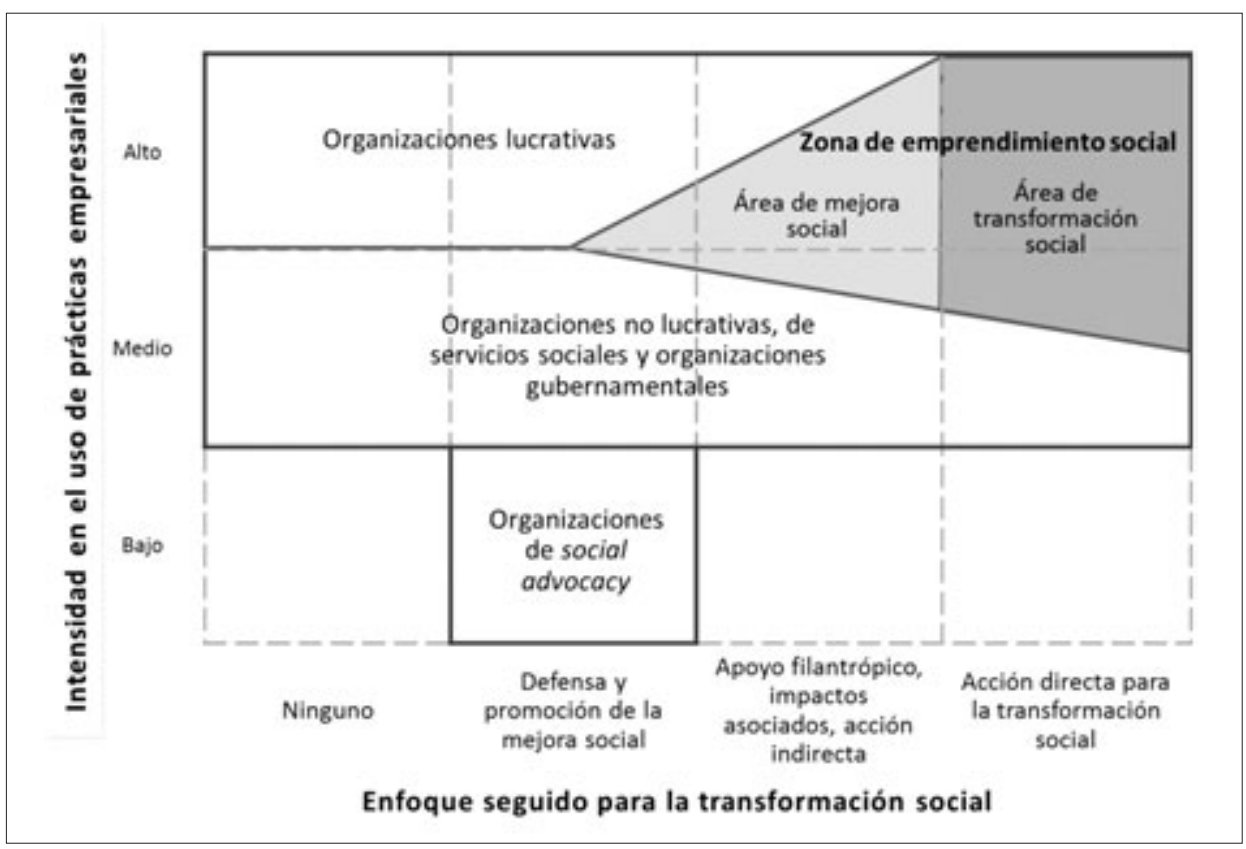

Fuente: Traducción propia de Swanson y Zhang (2010). 
De este modo, los autores arrojan luz sobre las borrosas fronteras existentes entre el emprendimiento social, el emprendimiento económico con resultados sociales, el sector público o el sector no lucrativo que utiliza estrategias de mercado para obtener financiación. La "zona de emprendimiento social" incluye, por tanto, a los emprendedores que actúan más allá del fin económico socialmente responsable:

1.- El "área de mejora social" representa a las organizaciones que tienen como parte del cumplimiento de su misión promover y facilitar el cambio social en otros agentes, por ejemplo, mediante la creación y comercialización de materiales educativos y de sensibilización sobre problemas sociales y ambientales. Tal es el caso de la empresa Ekilikuá, que entiende que los materiales que elabora "tienen como denominadores comunes la ilustración, la educación en valores, y un proceso productivo sostenible con las personas y con el medio ambiente" ?

2.- En el "área de transformación social", los emprendimientos no sólo inducen el cambio, sino que actúan de modo directo para la transformación social que define su misión, como ilustra el caso de SunnyMoney, una empresa creada por (y propiedad de) la ONG internacional SolarAid para distribuir a gran escala lámparas solares en África. La misión del "brazo comercial sin ánimo de lucro" de SunnyMoney es "erradicar las lámparas de queroseno de África para el final de esta década" ${ }^{8}$.

La energía que impulsa la creación y el funcionamiento de SunnyMoney es la inquietud ante la falta de acceso a electricidad que lleva a 600 millones de africanos a alumbrarse con lámparas de queroseno, con sus riesgos para la salud y la seguridad, sus contaminantes emisiones, su iluminación de bajo nivel y su elevado precio. Esa vía para luchar contra la pobreza y el cambio climático impulsó el nacimiento de SolarAid pero, como parte de su misión ajena al lucro, entendió que la lógica mercantil proporcionaba unas más amplias posibilidades de acción y de mantenimiento del servicio a lo largo del tiempo que la tradicional lógica de la caridad. Ésta se encuentra normalmente más restringida a programas de ayuda más puntuales y la sostenibilidad de su actividad y de su flujo de ingresos tiende a verse sometida a mayores dependencias y vaivenes coyunturales.

De ahí que todas las decisiones típicamente empresariales que SunnyMoney ha de tomar a lo largo de su cadena de valor, tales como el diseño del producto, la política de precios o la evaluación de los riesgos empresariales, tienen siempre una guía muy concreta: la voluntad de trabajar para luchar contra la pobreza y el

\footnotetext{
${ }^{7}$ Véase http://www.ekilikua.com/ekilikua/quienes/quienes.htm (acceso: 10/12/2013).

${ }^{8}$ Puede verse el detalle de su lógica de funcionamiento en http://www.solar-aid.org/how-it-works/ (acceso: 10/12/2013).
} 
cambio climático a través de la sustitución de lámparas de queroseno por una iluminación limpia, duradera y barata. En este sentido, es destacable el sistema de distribución único de SunnyMoney, como ejemplo del compromiso con la innovación, la adaptación y el aprendizaje permanentes que caracteriza al emprendimiento social para cumplir más eficazmente con su misión (Dees, 2001). En la implantación de su estrategia dirigida a construir el mercado de iluminación solar en África, se ha apoyado en las redes educativas, dada la legitimidad de la que éstas gozan, especialmente entre las comunidades más remotas y dependientes del queroseno. Así, en los mercados vírgenes, donde la demanda es muy baja o inexistente, trabajan con autoridades educativas locales para entrenar a los profesores en la promoción de lámparas solares entre los padres de los estudiantes. Conforme la demanda comienza a construirse y las personas experimentan los beneficios del uso de las lámparas, los directores de los colegios las venden directamente en sus comunidades. Finalmente, una vez que los primeros compradores ya se han consolidado, SunnyMoney desarrolla un ecosistema de distribuidores o agentes de ventas para hacer llegar las lámparas a una comunidad más amplia (Miller, 2013).

\section{LA EMPRESA SOCIAL COMO OPORTUNIDAD PARA AMPLIAR LA CONSCIENCIA SOBRE LA LÓGICA QUE SUSTENTA UN MODELO EN CRISIS.}

La natural deriva consumista que nuestro modelo económico en permanente expansión requiere para reproducirse conecta de un modo muy eficaz con la infinitud del deseo humano de bienestar hedónico (Riechmann, 2009), proporcionando numerosos beneficios materiales a grandes grupos de población desde hace siglos: elevados niveles de bienestar material, una vida más larga y saludable, la reducción de las barreras espaciales para la comunicación, una espectacular proliferación del conocimiento o un desarrollo tecnológico que hace parecer que todo fuera posible.

Ciertamente, como en cualquier otra burbuja, en la capitalista, a la par que ha ido creciendo en volumen y en extensión geográfica el número de individuos y colectivos que persiguen los beneficios que el modelo promete, también lo han hecho las tensiones entre la racionalidad que rige en su interior y la que subyace a una realidad más amplia (Senge et al., 2009). Tensiones que adquieren una forma evidente en los mencionados límites biosféricos, la desigual distribución de beneficios y costes asociados al sistema, el empobrecimiento de la vida comunitaria, alienaciones y exclusiones sociales o el acaecimiento de crisis económicas periódicas. En definitiva, las tensiones propias de un proceso de "acumulación por desposesión”, según la expresión acuñada por Harvey (2010). La lógica dominante en el interior de la burbuja es asumida de modo generalmente acrítico, condicionando las decisiones que las empresas tienden a adoptar en el ejercicio coti- 
diano y estratégico de su actividad. Por ello, las empresas sociales constituirían una manera de experimentar en la práctica el cuestionamiento de un modelo de pensamiento ampliamente seguido en la arena empresarial. Esa experimentación facilita que, a su vez, otros agentes (en tanto socios, clientes, reguladores, proveedores o financiadores) puedan poner en duda sus propias creencias automatizadas que, siguiendo a Naredo (2006), podrían sintetizarse del siguiente modo:

1. La interpretación del proceso económico como un proceso de generación de riqueza expresada en términos monetarios y el correspondiente uso del razonamiento monetario como guía suprema de gestión. La misión social de las empresas sociales busca trascender el grave reduccionismo que este principio supone, dado que subordina a la económica las dimensiones física y moral subyacentes. Además, al homogeneizar todo el proceso en un único tipo de valor, el monetario, se ocultan (o se dificulta su visualización y consideración) los deterioros físicos y sociales generados en el mismo. Sirva como ejemplo del cuestionamiento de este principio el caso de Milhistorias, una empresa de inserción promovida por Rais Fundación para crear puestos de empleo protegido mediante la actividad de comercialización de productos procedentes de la agricultura ecológica9. El razonamiento moral (trascendiendo el criterio monetario como base de la concepción del trabajo y su valoración) y el físico (con el fomento de productos de la agricultura ecológica) están en la esencia de su actividad económica.

2. La interpretación anterior, al resaltar la idea positiva de riqueza asociada al proceso económico, sirve de soporte a otra asunción: el crecimiento económico como un fin intrínsecamente bueno para todo el mundo. "El crecimiento del consabido agregado monetario de producto o renta nacional se percibe como algo inequívocamente deseable y generalizable, sin necesidad ya de analizar su contenido efectivo, sus servidumbres y sus consecuencias no deseadas" (Naredo, 2006: 67). Sin embargo, si una empresa social tiene vocación de crecimiento, ésta se verá más bien ligada al deseo de ampliar el alcance de la transformación social que busca, como ejemplifica SunnyMoney y su misión de erradicar del continente africano las lámparas de queroseno y sustituirlas por iluminación basada en la energía del sol.

3. Todo lo anterior privilegia el registro del coste de extracción sin incluir el de reposición (entendamos extracción y reposición en sentido no sólo físico, sino también social). A su vez, esto provoca una asimetría que sesga la valoración del proceso hacia las fases finales de comercialización, que se llevan la parte mayoritaria de la "creación de valor" frente a las fases de extracción y tratamiento. El cuestionamiento de este principio constituye el eje central de la actividad de emprendimiento social centrada en el comercio justo, en su objetivo de

\footnotetext{
${ }^{9}$ Véase http://www.milhistorias.es/milhistorias-proyecto-social (acceso: 30/10/13).
} 
reconocer, visibilizar y valorar la fase de extracción. Por ejemplo, Cafédirect es una empresa británica de café, té y chocolate que busca resolver los mencionados sesgos de la valoración económica trabajando directamente con los cultivadores y compartiendo con ellos decisiones y beneficios ${ }^{10}$.

Esos principios producen un mundo teóricamente coherente pero autodestructivo en la práctica, a la luz de las evidencias de insostenibilidad global. Un mundo que asume que la realidad del comportamiento humano es la representada por el modelo simplificado del homo oeconomicus. Una teorización que privilegia la dimensión egoísta de un ser humano que expresa sus deseos fundamentalmente a través de los mercados y que racionaliza sus decisiones maximizando su utilidad individual. Un homo que prefiere tener a ser, como ya nos recordaba Fromm (1980) cuando distinguía la debilidad de una identidad basada en las posesiones frente a otra mucho más liberadora basada en el ser.

Pero, efectivamente, el comportamiento humano no es sólo (o es mucho más que) egoísmo, pensamiento eminentemente racional y optimización de la utilidad individual. Su dimensión social, su capacidad de empatía y de compasión, así como su deseo de trascendencia se expresan constantemente a través de comportamientos altruistas y de la orientación al cuidado de otros seres humanos, de seres no humanos y de la Tierra como un todo (Cavagnaro y Curiel, 2012). La empatía y la compasión (por otros seres más o menos distantes o por todo el conjunto de la biosfera) movilizan, en última instancia, la consciencia de la que nace la empresa social. Por tanto, como detallaremos más adelante, el emprendimiento social estaría contribuyendo a dar relevancia a dimensiones del ser humano tradicionalmente marginadas por el modelo capitalista.

\section{LA EMPRESA SOCIAL COMO EJERCICIO DE APRENDIZAJE EXPERIENCIAL EN EL MARCO DE LAS TRANSICIONES A LA SOSTENIBILIDAD.}

Cuestionar esos principios largamente arraigados es un reto, no sólo por la duración centenaria del modelo capitalista, sino también porque el cambio de mirada requiere una perspectiva sistémica, compleja e integradora, lo que resulta incompatible con el actual dominio de la especialización y el pensamiento racional. Un dominio que Descartes (1982 [1637]: 51) ilustra perfectamente en el segundo paso de su método: "Dividir cada una de las dificultades que encontrase en tantas partes como pudiera y fuere necesario para resolverlas mejor". En este sentido, Morin (1993: 69) cuestiona una concepción de lo científico caracterizado por esa "falsa racionalidad" y reivindica

\footnotetext{
${ }^{10}$ Véase http://www.cafedirect.co.uk/smallstory/ (acceso: 9/12/2013).
} 
el conocimiento desde la integración de lo que está separado y desconectado: "La inteligencia parcelada, compartimentada, mecanicista, disyuntiva, reduccionista, rompe lo complejo del mundo en fragmentos disjuntos, fracciona los problemas, separa lo que está enlazado, unidimensionaliza lo multidimensional. Es una inteligencia a la vez miope, présbita, daltónica y tuerta; lo más habitual es que acabe ciega (...). Incapaz de enfocar el contexto y el complejo planetario, la inteligencia ciega se vuelve inconsciente e irresponsable".

Ante la necesidad de esa mirada holística y sistémica a la que apelan las evidencias de una crisis ecosocial global, a continuación se aborda la idea de sostenibilidad como un paradigma más adecuado, y a la empresa social como un ejercicio de aplicación práctica de este paradigma en el contexto empresarial.

\subsection{Superando la retórica del "sosteniblablá".}

Abordar los desafíos de la crisis ecosocial requiere considerar el modelo de funcionamiento de la biosfera como una referencia ineludible y, con él, un cambio de foco: en las conexiones más que en las partes; en los procesos circulares y cerrados más que lineales y abiertos; o en la perdurabilidad y la renovabilidad más que en la obsolescencia y el residuo. De ahí que la idea de sostenibilidad global haya venido tomando protagonismo como un constructo que representa un nuevo paradigma. Efectivamente, vivimos en un planeta finito, marcado por complejas interconexiones y por procesos de degradación entrópica. Por tanto, su sostenibilidad requiere mantener la integridad de sus procesos, ciclos y ritmos. Y ese respeto significa también el desplazamiento, desde la asunción del infinito necesitar/desear consumista del ser humano, a su también realidad compasiva centrada en el cuidar como condición básica para la perdurabilidad y una vida buena.

Sin embargo, no podemos olvidar que la idea de sostenibilidad también ha sido apropiada e instrumentalizada por el modo de funcionamiento capitalista para, de nuevo, eludir los mencionados límites físicos y sociales a su expansión. Así, podemos observar cada día multitud de propuestas de "productos sostenibles" y su correspondiente "consumo sostenible", "políticas y estrategias de sostenibilidad" o "informes de sostenibilidad" que tienden a banalizar el término convirtiéndolo esencialmente en "sosteniblablá" (como versión en español del término "sustainababble" denunciado por Engelman, 2013). Una retórica que, en el mejor de los casos, supone un menor daño que otra alternativa de ese producto/política/ estrategia/práctica pero que, muy raramente, implica un desplazamiento hacia el ser y el cuidar en términos individuales, sociales y biosféricos ${ }^{11}$.

${ }^{11}$ El mal uso y el abuso del término sostenibilidad resulta ilustrativamente explicado en las palabras de Ehrenfeld y Hoffman (2013: 15): "It's misused when those who speak it and act in its name do not understand what it means. It's abused when it is used by agents that know they do not understand it, but use it as an attempt to fool others into thinking they do". 
Y, en esencia, al incorporarse el discurso de la sostenibilidad a la racionalidad que sirve de soporte al capitalismo y al dominio de las relaciones mercantiles en todos los órdenes vitales, el "sosteniblabá" no tiene capacidad real de cuestionar dicha racionalidad (Ehrenfeld y Hoffman, 2013). Así, sin un rediseño de los sistemas socio-técnicos, que siguen respondiendo de modo prioritario a la lógica del mercado, del beneficio y del rendimiento sobre la inversión, la normalización del término sostenibilidad en el discurso empresarial significa la continuidad del modelo en la práctica.

Efectivamente, la respuesta a la insostenibilidad va muchísimo más allá de la elección de tecnologías más eficientes en el consumo de energía, de la adquisición de productos de comercio justo o de la instalación de guarderías en los centros de trabajo, por ejemplo. Se trata de un reto que, como advierten Hirsch Hadorn et al. (2008), requiere capturar la complejidad de los problemas y las diversas visiones de los mismos, construyendo conocimiento con el foco puesto en su resolución para lo que se entiende que es el bien común. En este sentido, Harvey (2010) sigue un enfoque coevolucionario ${ }^{12}$ para describir el avance del capitalismo como la interacción entre seis esferas: concepciones mentales del mundo; reproducción de la vida cotidiana; tecnologías y formas organizacionales; comportamiento humano y relaciones sociales; procesos productivos y laborales; mecanismos institucionales; y relaciones con la naturaleza.

Pero las perspectivas holísticas superadoras del "sosteniblablá" requieren un pensamiento complejo, en el sentido planteado por Morin (1993), que permita trascender las barreras entre diferentes disciplinas y tradiciones académicas. De ahí que el territorio de las transiciones de los sistemas socio-técnicos hacia la sostenibilidad esté comenzando a constituirse como campo de investigación específico. Las transiciones son procesos de transformación social radical, en los que los sistemas cambian de modo estructural a lo largo de un período de tiempo e implican la transformación de las interacciones entre diversos grupos sociales, incluyendo empresas, usuarios, comunidad científica, políticos, movimientos sociales y grupos con intereses especiales (Geels y Schot, 2010; Rotmans et al., 2001). La transición a la sostenibilidad implica la revisión de las creencias y significados culturales en los que se sustenta el funcionamiento de nuestros sistemas socio-técnicos, incluyendo dimensiones como tecnología, regulación, prácticas de los usuarios, mercados, infraestructuras, redes de mantenimiento y redes de aprovisionamiento. Estas complejas dinámicas pueden ser explicadas en tres niveles (Geels 2004):

12 Norgaard (1994) toma prestado de la biología el término coevolución en referencia al cambio evolutivo recíproco de dos especies en estrecha interacción. Explica, así, el "desarrollo" como un proceso en coevolución entre conocimiento, valores, organización, tecnología y medio ambiente. 
1.- Régimen socio-técnico, como configuración dominante de ciertos artefactos tecnológicos, prácticas de los usuarios, estructuras de mercado, marcos reguladores, valores culturales y conocimiento científico.

2.- Territorio socio-técnico, referido a las dimensiones espaciales externas, fuera del control de los actores, que cambian lentamente y que resultan más difíciles de modificar (caso de las infraestructuras, el urbanismo o los problemas ambientales acumulativos).

3.- Nicho tecnológico, donde se producen las innovaciones radicales, frente a las incrementales de los anteriores niveles. Pueden romper la situación dominante cuando los correspondientes procesos que tienen lugar en los niveles de régimen y territorio son favorables para crear una ventana de oportunidad. Una vez que la innovación en el nivel del nicho tecnológico se abre paso en la sociedad de modo masivo, entra en competencia con el régimen dominante y, eventualmente, puede reemplazarlo, introduciendo cambios más amplios en dimensiones como las infraestructuras, los valores o la regulación.

Las empresas sociales suponen un ejemplo de innovación dentro del nivel de nicho tecnológico, dado que rompen con el pensamiento dominante del negocio definido de modo primario por un fin económico. Y cabe la posibilidad de que entren en competencia con él, al menos en determinados sectores de actividad. Pensemos, por ejemplo, en el caso de Som Energia, una empresa cooperativa comercializadora de electricidad que tiene como objetivo cambiar el modelo energético actual a partir del consumo y producción de energía de origen renovable ${ }^{13}$. Además de su misión orientada a llevar a la práctica la provisión de electricidad a partir de fuentes enteramente renovables, su forma jurídica de cooperativa ya supone una diferencia con respecto al modelo dominante de provisión y consumo de electricidad: la implicación de los usuarios en la empresa va mucho más allá del acto de consumo, dado que su condición de socios (y, eventualmente, de proveedores de energía) les hace partícipes del proceso para el cambio que busca la empresa. Tal implicación, sin duda, favorece también la transición en el nivel del régimen socio-técnico, dado que la expansión de la base de socios lleva aparejada una más amplia transformación en las creencias y en las prácticas sobre producción y consumo energético ${ }^{14}$.

13 Véase http://www.somenergia.coop/es/ (acceso: 10/12/2013)

${ }^{14}$ Tras sus dos años de actividad, la cooperativa integra 11.301 socios y, desde su origen centrado en Cataluña, está expandiendo su presencia en otras regiones a través de grupos locales. 


\subsection{La empresa social y la oportunidad de los enfoques pragmáticos para la sostenibilidad.}

Lo anterior nos conduce a plantear que las transiciones a la sostenibilidad como campo de investigación presenta claros vínculos con la tradición filosófica pragmatista. Para el pragmatismo, la relevancia del significado de un pensamiento es la conducta que el pensamiento puede producir, de modo que el foco se pone en el proceso de aprendizaje, interpretación, experimentación y transformación de una comunidad, más que en un movimiento que tiene por objeto hacer corresponder determinadas ideas con una realidad externa (Ehrenfeld y Hoffman, 2013; Holden, 2008). En palabras de Maturana y Varela (1987: 27), desde la perspectiva de las raíces biológicas de la cognición humana, "all doing is knowing and all knowing is doing", es decir, el mundo que llegamos a conocer a través de la experiencia es el mundo que creamos a través de nuestro conocimiento. Según Levin y Greenwood (2001), este enfoque vendría a aliviar sesgos de la tradición investigadora dominante, marcada por la tendencia a elegir las cuestiones teóricas y de investigación desde la aplicación de normas internas de la profesión, dando más importancia al consenso (y el control) de la comunidad profesional que al carácter holístico de las situaciones y a la búsqueda de conocimiento verdaderamente útil para la realidad social.

Las experiencias de emprendimiento social constituyen un valioso ejercicio de experimentación. Su estudio aporta precisamente la utilidad de probar el funcionamiento en la práctica de una solución empresarial a los retos de la crisis ecosocial, facilitando el aprendizaje de vías para transitar el camino a la sostenibilidad ${ }^{15}$. De hecho, la aproximación pragmática invita a evaluar una idea como el emprendimiento social y su eficacia desde el criterio pragmático de verdad. Se trata de un proceso indisolublemente vinculado a nuestra experiencia que analiza cada idea a través de sus consecuencias prácticas, es decir, considerando las diferencias que generará que esa idea sea verdadera o no (James, 2008). Este enfoque se encuentra en el origen de las empresas sociales creadas por la conocida iniciativa de Muhammad Yunus para generar impactos positivos en salud, alimentación, creación de empleo, acceso a agua potable o financiación: "como cualquier idea nueva, el concepto de empresa social necesita ser demostrado en la práctica. Por eso he comenzado a crear empresas sociales en Bangladés" (Yunus, 2011: 21).

\footnotetext{
${ }^{15}$ Aunque en el campo de la ética ambiental existe una aparente pugna entre las estrategias pragmáticas -centradas en las respuestas prácticas a los problemas ambientales-y cosmológicas -centradas en el cambio de valores ambientales-, estamos de acuerdo con la literatura que ve una falsa disyuntiva, por cuanto las propuestas de cambio en la gestión van de la mano de cambios culturales (véase, por ejemplo, Ehrenfeld y Hoffman, 2013; Jenkins, 2011; Holden, 2008; y Minteer, 2008).
} 
Es también ilustrativo en este sentido el estudio que Ashoka realiza anualmente sobre la actividad de su red global de emprendimientos sociales, a través de encuestas y entrevistas realizadas a una muestra de emprendedores de 42 países que habían sido seleccionados por la organización hace cinco años. Los resultados muestran que en ese plazo el 54\% de los encuestados había cambiado sistemas de mercado a escala nacional; el 57\% había conseguido modificaciones en políticas públicas (cambios legislativos, organización de la acción ciudadana o cambios en códigos de conducta y normas sectoriales); el 54\% había trabajado en la integración social de grupos marginales; y una proporción similar consideraba la empatía como el factor más importante de su labor (Ashoka, 2013). Aunque cabe advertir que se incluye una noción de emprendimiento social más amplia que la que empleamos en este trabajo, tales datos constituyen un buen ejemplo del papel que el emprendimiento social puede ejercer en las transiciones a la sostenibilidad.

A escala europea, el reciente informe de la Comisión Europea (2013:49) sobre economía social y emprendimiento social pone de manifiesto que las empresas sociales generan un notable impacto social, consecuencia directa de su misión social pero también de su estructura: generalmente basadas en la reciprocidad y en la cooperación, la implicación de sus distintos públicos en su gobierno asegura que la organización actúa de modo más fiel a los intereses de sus miembros y su comunidad, ayudando a incrementar el capital social. Además, el consorcio de investigación de ámbito europeo SELUSI (2010), a partir de la creación y análisis de una base de datos de más 550 empresas con fin social de España, Hungría, Reino Unido, Rumanía y Suecia, obtiene entre sus resultados que las empresas sociales mantienen una actividad innovadora notablemente superior a las empresas convencionales. Una innovación motivada de modo mayoritario por el deseo de mejorar la manera de producir impacto social y/o de aumentar su alcance incrementando el número de personas a las que llega su impacto.

Con objeto de ampliar la capacidad de atender a los retos sociales que este tipo de empresas lleva en su razón de ser, plataformas europeas de cooperación como la European Social Franchising Network (ESFN) o el International Centre for Social Franchising invierten en el desarrollo de la franquicia social compartiendo conocimiento, identificando modelos de franquicia y ayudando a empresas sociales a desarrollarla, contribuyendo así a replicar y escalar estos modelos (Comisión Europea, 2013). Como un ejemplo de su impacto social, la ESFN encuentra que las más de 60 franquicias sociales existentes en Europa han creado al menos 10.000 empleos en la economía social, $65 \%$ de los cuales presentan algún tipo de desventaja en el mercado laboral convencional (Bartilson, 2012). 


\section{LA EMPRESA SOCIAL COMO UN TERRENO PROPICIO PARA EL AVANCE DE LA DEMOCRACIA Y LA RESPONSABILIDAD EN EL GOBIERNO DE LA EMPRESA.}

Como advertíamos antes, el uso del término sostenibilidad es con frecuencia instrumentalizado y convertido en "sosteniblablá" con objeto de sortear los límites a la expansión del capital, obstaculizando así su cuestionamiento. El hecho de que la empresa se haya configurado como agente protagonista de ese proceso económico y motor esencial de la dinámica capitalista, nos remite a la pregunta que Handy (2002) se hacía al calor de los escándalos que protagonizaba el panorama corporativo a principios del presente siglo: ¿para quién y para qué la empresa?

Aunque las diversas aportaciones de las teorías económicas de la empresa han venido introduciendo perspectivas complementarias para responder a esta pregunta ${ }^{16}$, el enfoque neoclásico de la empresa como una función de producción, como una "caja negra" cuyo objetivo es maximizar beneficios o minimizar costes, sigue siendo dominante en la esencia del funcionamiento empresarial. Una máquina optimizadora de utilidad, fiel reflejo del comportamiento del homo oconomicus que la crea, la dirige y la hace funcionar. Una máquina que, ante las tensiones asociadas al modelo de pensamiento que la sostiene, puede mantener un discurso completamente disociado de su acción. Así, puede autodenominarse sostenible, por ejemplo, por incrementar la eficiencia en el consumo unitario de un recurso pero, dados los condicionantes de la dinámica expansiva capitalista, muy probablemente estimulará el sobreconsumo de sus productos, con el consiguiente aumento absoluto del recurso inicialmente ahorrado. Ciertamente, este ejemplo se presenta de modo pertinaz en muchas dinámicas empresariales supuestamente "sostenibles": a pesar de los continuos avances en la eficiencia de los procesos productivos, el requerimiento total de materiales medio per cápita en el mundo no ha cesado de aumentar (Naredo, 2006). Ello, en buena medida, es debido al "efecto rebote" que con frecuencia ha venido aparejado a las "salvadoras" tecnologías ecoeficientes, y que consiste en aumentar el consumo total como consecuencia del ahorro económico y la conciencia de un consumo más eficiente (Riechmann et al., 2012).

Pero iqué incentivos podrían romper con esa dinámica expansiva y facilitar el camino hacia un "para qué" y un "para quién" más alineados con la sostenibilidad

\footnotetext{
${ }^{16}$ Como una estructura de gobierno alternativa al mercado que tendrá como objetivo básico economizar los costes de transacción; como una red de contratos incompletos, marcados por unas relaciones de agencia que implican asimetrías en el acceso a la información de los actores involucrados y consecuentes costes de control y garantía; o como resultado de rutinas organizativas que, junto con los recursos y capacidades desarrollados a lo largo de la historia de la empresa, constituyen su esencia y la fuente básica de su ventaja competitiva (véase, por ejemplo, la síntesis de Valdaliso y López, 2000).
} 
global? Una línea de respuesta se encuentra en la profunda transformación del concepto de propiedad de la empresa derivada de la evolución de los mercados de capitales. Esta transformación cuestiona las bases teóricas dominantes del gobierno de la empresa y, con ello, de la consideración del poder en la empresa y del ejercicio de su responsabilidad social.

\subsection{La propiedad del "valor" de la empresa ya no es lo que era.}

El trabajo clásico de Barle y Means ya planteaba en 1932 la necesidad de revisar la consideración de la propiedad de la empresa y su gobierno a la luz de la creciente liquidez de los mercados de capitales. Y la vigencia de sus postulados se ve intensificada en la actualidad por el dominante papel de las instituciones de inversión colectiva y, en general, por el proceso de financiarización de la economía que ha protagonizado las últimas décadas (Aglietta y Rebérioux, 2005).

Efectivamente, las bases de la empresa han cambiado en la medida en la que la propiedad se ha visto sustituida por la inversión y que los verdaderos activos de la empresa se encuentran de un modo creciente en las personas (Handy, 2002) y en las capacidades colectivas creadas por la organización, mucho más que en los activos objeto de propiedad que figuran en el balance de la compañía. Aunque las diferencias sobre la generación y el destino del beneficio son claras en el caso de las empresas cotizadas, con propiedad y control claramente separados (managerial), frente a empresas individuales o no cotizadas (entrepreneurial), si algo caracteriza al proceso de creación de valor de la empresa es su naturaleza colectiva y la multiplicidad de su origen (Ghoshal, 2005).

Aceptar que el valor creado es fruto de la intervención coordinada de recursos tangibles e intangibles y de capacidades desarrolladas por el colectivo que forma la organización, hace difícil seguir defendiendo que el accionista sea jerárquicamente superior a los demás constituyentes de la empresa, idea tradicionalmente sustentada en los argumentos del beneficio y del riesgo. Por un lado, el hecho de que la empresa genere beneficio no quiere decir que deba ser reservado para remunerar de modo exclusivo o prioritario a los accionistas, porque no es el único que participa en su creación. Por otro, el capitalismo financiero transfiere el riesgo del accionista a toda la sociedad. En coherencia con la decadencia de tales argumentos, la dirección debería orientarse a satisfacer los intereses de la entidad misma, trascendiendo los intereses de un stakeholder prioritario. Esta orientación facilitaría la introducción de comportamientos más coherentes la idea de sostenibililidad global.

A la luz de tales transformaciones, Berle y Means hacían un llamamiento a la redefinición del poder en la empresa, que habría de ser ejercido desde bases más democráticas en nombre del colectivo que es la propia organización, no en el de uno de sus stakeholders. Sin embargo, la recomendación de estos autores quedó 
relegada bajo el dominio de la teoría contractual, reafirmándose así la soberanía del accionista (Aglietta y Rebérioux, 2005).

El predominio del valor del accionista, junto con la evolución que han experimentado los procesos de expansión capitalista y su forma de eludir los límites a su acumulación, ha favorecido la existencia de formatos empresariales que acumulan ingentes cantidades de poder a escala global. Poder que es gestionado desde criterios financieros en última instancia y, con frecuencia, concentrado para servir a la generación de rentas para una élite directiva (integrada por una red de directivos, banqueros de inversión y socios de grandes firmas de abogacía y consultoría) al frente de los consejos de administración de estas corporaciones "financiarizadas" (Harvey 2010).

\subsection{Desde el poder a la vocación social en el gobierno y la responsabilidad empresariales.}

Ante el anterior contexto, Bendell (2004) identifica las respuestas que han venido dándose al creciente poder corporativo y que dependen de cómo este poder sea percibido, tal y como recogemos en el Cuadro 1.

\section{Cuadro 1. Percepciones del poder corporativo}

\begin{tabular}{|c|c|}
\hline PERSPECTIVA DEL PODER & RESPUESTA \\
\hline $\begin{array}{l}\text { Como oportunidad, potenciando los impactos } \\
\text { positivos de las corporaciones sobre la sociedad. }\end{array}$ & $\begin{array}{l}\text { Responsabilidad social corporativa, ciudadanía } \\
\text { corporativa o filantropía corporativa. }\end{array}$ \\
\hline $\begin{array}{l}\text { Como obstáculo para que otros agentes puedan } \\
\text { desarrollar sus objetivos sociales o ambientales. }\end{array}$ & $\begin{array}{l}\text { Movimiento por la corporate accountability, para } \\
\text { promover la capacidad de aquéllos afectados por } \\
\text { una corporación para regular sus actividades. }\end{array}$ \\
\hline $\begin{array}{l}\text { Como obstrucción para superar la lógica sistémica } \\
\text { de la acumulación de capital. }\end{array}$ & \multirow{2}{*}{$\begin{array}{l}\text { Cuestionamiento del poder corporativo y de } \\
\text { la propia corporación: de su definición, de su } \\
\text { forma de proceder y de su papel en la sociedad. }\end{array}$} \\
\hline $\begin{array}{l}\text { Como problema moral, ya que las instituciones } \\
\text { poderosas deberían ser, por principio, democráticas } \\
\text { o controladas por medios democráticos. }\end{array}$ & \\
\hline
\end{tabular}

Fuente: Adaptado de Bendell (2004).

La visión del poder corporativo como oportunidad conduce al desarrollo de la RSC como una respuesta que destaca los potenciales impactos sociales positivos que su amplia capacidad de acción posee. De ahí que no rechace ni cuestione el poder de la corporación, pero reconoce que también tiene una responsabilidad por sus impactos sociales y ambientales más allá de la responsabilidad legal, así como por el comportamiento de otros agentes con los que interactúa (por ejemplo, en las cadenas 
de aprovisionamiento). En definitiva, apela a la necesidad de que las empresas gestionen su relación con la sociedad, ya sea por razones de viabilidad comercial o para generar valor social (Blowfield y Murray, 2011; Blowfield y Frynas, 2005).

La inclusión voluntaria de estándares y prácticas éticas más allá de las reglas del juego básicas impuestas por la legislación y la norma de la industria puede estar más o menos integrada en el corazón del modelo de la empresa. Así, la práctica de la RSC muestra una gama de formatos más alineados con la obligación moral y la esencia de la actividad de la empresa, o más marginales e instrumentales para el fin último de la rentabilidad económica. Sin embargo, la RSC no lleva en su definición la necesidad de que la empresa esté dirigida por una misión social (Comisión Europea, 2013), ni tampoco está diseñada para poner en cuestión las bases de la civilización industrial y su modelo de producción y consumo capitalista.

En definitiva, la RSC implica avances en la consciencia sobre el daño ambiental y social de la actividad empresarial, así como sobre las limitaciones de la capacidad reguladora del mercado y del Estado (Utting, 2005). Pero esto supone trasladar capacidad de regulación sobre aspectos relacionados con el bien común a agentes cuyo interés no está necesariamente alineado con tal fin último, cuestión que se pone más claramente de manifiesto cuando la empresa opera en contextos de extrema pobreza, conflictos armados, regímenes opresivos o paraísos fiscales.

En consecuencia, la RSC se centra sólo en aquellos aspectos empresariales que se entienden negociables dentro de este modelo. Da a unas compañías gobernadas desde bases no democráticas ${ }^{17}$ el poder de decidir lo que significa ser responsable, lo que obstaculiza la reflexión sobre el poder corporativo y su cuestionamiento (Corporate Watch, 2006) y, en general, sobre los principios del funcionamiento del capitalismo.

La perspectiva del corporate accountability trata de suplir tales limitaciones: en vez de apelar a las compañías para que den cuenta de sus actividades e impactos de modo voluntario, y para que mejoren su desempeño social y ambiental también de modo voluntario (lo que tenderá a ocurrir desde una guía económica, no moral), el movimiento por la accountability implica (Bendell, 2004): 1) obligación legal de responder por sus decisiones, acciones y resultados; 2) sancionabilidad del incumplimiento; y 3) estándares que se aplican de modo mucho más amplio que sólo a las empresas que eligen adoptar iniciativas voluntarias.

${ }^{17}$ En el sentido que señalábamos anteriormente y que, en esencia, entiende a la corporación como la propiedad de sus accionistas. Accionistas que, con la evolución de los mercados financieros, han cambiado el control de la empresa por la liquidez de su inversión, cediendo ese control a los agentes que dirigen las corporaciones. Por tanto, se ignora, en la práctica, la naturaleza colectiva de la creación de valor empresarial y al resto de participantes en el proceso. 


\subsection{La misión social de la empresa como base para un gobierno empresarial más democrático y responsable.}

La idea de empresa social, puesto que está diseñada desde la búsqueda de la contribución al bien común, constituye un terreno propicio en el que hacer más visible la naturaleza colectiva de la creación de valor en la empresa y, en coherencia, ejercitar la práctica del gobierno de la empresa desde principios más democráticos (tal y como vimos con el ejemplo de Som Energia). Es cierto que el cumplimiento de su misión social supondrá que, en la práctica, muchas empresas sociales puedan privilegiar la atención a uno o varios colectivos/problemas. Sin embargo, esto será una consecuencia del cumplimiento de su misión social, pero no constituirá el criterio que articula su proceso de creación de valor. Dicho de otro modo, es la consciencia sobre un problema social la materia prima con la que se define el valor que crea la empresa.

Ahora bien, la ejecución práctica de ese proceso no está exenta de sesgos y contradicciones que pueden acompañar a los logros relevantes que la empresa social consigue en la transformación social que acomete. Por ejemplo, una empresa puede estar logrando importantes avances en la garantía de un precio justo y estable para proveedores de productos de comercio justo, o en la creación de puestos de trabajo para personas con riesgo de exclusión laboral. Y, simultáneamente, esa actividad puede estar ocasionando otros impactos negativos que no son contemplados en el proceso de creación de valor, por ejemplo, derivados del transporte a largas distancias de productos de comercio justo frente al aprovisionamiento local, o de un mayor trasiego de recursos escasos.

Pero aquí cabe insistir en esa consciencia que fundamenta el emprendimiento social, ya que pone de manifiesto una visión del ser humano mucho más compleja que la definida por el homo œeconomicus. Efectivamente, de acuerdo con Cavagnaro y Curiel (2012), el desarrollo humano puede interpretarse como un proceso de reducción del egocentrismo y de expansión de la conciencia y la compasión:

1.- El paradigma económico convencional sesga al ser humano hacia la dimensión ligada al individualismo y a la inteligencia racional ("yo"). Son aspectos ciertamente arraigados en nosotros porque se asientan en nuestra urgencia para sobrevivir, algo compartido con todos los seres vivos.

2.- Por otro lado, la dimensión "yo y tú", aunque no forme parte esencial del homo oeconomicus, apela a la naturaleza social del ser humano: ha ido aprendiendo a lo largo de su historia que la cooperación con otros hace posible alcanzar resultados que uno no puede lograr en solitario. Entonces, la empatía y la lealtad hacia el grupo se fortalecen.

3.- Por último, la dimensión "todos y todo" hace referencia a valores biosféricos conectados con la compasión por todos los seres vivos y la Naturaleza como 
un todo. La compasión, como destaca la ecología profunda (Næss, 1995), está en la base de la experiencia de un "yo" ampliado que implica una percepción de estar relacionado con (o ser idéntico a) otras formas de vida; un "yo" que se traduce en un comportamiento espontáneo con la ecosfera (y los seres que la integran) como nos comportaríamos con nosotros mismos.

De lo anterior se desprende que la inquietud por un problema social (en sentido amplio) y la energía para cambiarlo, como pilares que sustentan el emprendimiento social, sólo pueden nacer desde el desarrollo de las tres dimensiones. Si bien las dos primeras se basan en formas de inteligencia (racional y social) entrenadas con profusión en cualquier tipo de emprendimiento, la compasión emerge de un tipo de inteligencia (espiritual) menos destacada y menos practicada por el modelo económico dominante. Sin embargo, cabe aceptar con Parkin (2010) que la compasión por todos y todo es el combustible que estaría alimentando el liderazgo para la sostenibilidad y, como parte de él, el emprendimiento social.

En definitiva, si en la sección previa defendíamos el emprendimiento social como un territorio fértil para el aprendizaje y la experimentación de formas empresariales innovadoras para las transiciones a la sostenibilidad, reafirmamos aquí su utilidad para avanzar en la discusión sobre la democracia en la empresa y el ejercicio de su responsabilidad. La empresa social visibiliza una experiencia que contrasta con el modelo que muestra el emprendimiento económico, debido a que emerge desde una comprensión profunda y compasiva de la biosfera y de la actividad humana en ella.

\section{CONCLUSIONES.}

El modelo de producción y consumo capitalista está marcado por una paradoja: los múltiples beneficios materiales que es capaz de generar han hecho dominar la convicción de que se trata del mejor de los sistemas posibles. Sin embargo, la dinámica de acumulación expansiva que define el modelo ignora los límites de la capacidad de la Tierra para renovar sus procesos y, en general, las bases de una vida buena que no puedan entrar en la dinámica mercantil convencional. Como resultado, acarrea una pesada mochila de desequilibrios ambientales y sociales.

La centenaria duración del funcionamiento del modelo, junto con su capacidad para ir sorteando los límites a su expansión (no sin duros ajustes en forma de crisis periódicas, más o menos globales), dificultan el enjuiciamiento de conceptos y asunciones propios del sistema, que han ido incorporándose acríticamente a nuestros esquemas de pensamiento y orientando nuestra visión del mundo, tales como la fe en un "progreso" material ilimitado, el imperativo tecnológico o la mitología del desarrollo. En consecuencia, nos encontramos siguiendo lógicas individualmente racionales que se traducen en elecciones colectivamente irracionales. 
En este marco, la empresa social constituye un campo de investigación interesante por la combinación atípica de características que la adornan. Se distancia de la empresa (y de la forma de hacer empresa) convencional porque no nace guiada por un fin económico, sino por la consciencia de desequilibrios sistémicos, por la voluntad de paliar las negativas consecuencias generadas y, en última instancia, por el deseo de transformar el sistema. Su misión social inspiraría todas las decisiones de su proceso de creación de valor, incluyendo su vocación de perdurabilidad para dar respuesta al problema social que la mueve.

Pero servir a su misión social no significa renunciar a la potencia del mercado para ampliar su alcance y para generar una fuente de ingresos con visos de estabilidad; tampoco significa renunciar al uso de prácticas y herramientas empresariales que se han mostrado útiles para capacitar a un colectivo en el logro de una misión de modo eficiente y eficaz, si bien esas herramientas se aplican del modo innovador que el fin social estimula.

El rechazo al uso del mercado es más dominante en el sector no lucrativo tradicional (ya que el pago de un precio se aleja de la idea de regalo, expresión más verdadera de la caridad). Sin embargo, la empresa social sí utiliza estrategias de mercado, pero incorporando a la vez el típico impulso creador de las organizaciones no lucrativas: aquél basado en una consciencia más amplia sobre el papel del ser humano en la biosfera y en su relación con otros seres humanos y no humanos. Un impulso que nace de la dimensión empática y compasiva de la persona, más que del egoísmo y el individualismo.

Así, la empresa social pone de manifiesto la debilidad de las sólidas fronteras que tradicionalmente han separado al sector lucrativo y del no lucrativo. Deja estéril la disyuntiva lucro-no lucro para poner en el centro de su ser la comprensión de un problema social y su voluntad de cambio. Y ello usando un lenguaje propio del territorio empresarial, fácilmente comprensible por el modelo de pensamiento dominante. Pero, como es también un idioma que surge desde una visión más sistémica y desde la compasión como valor básico, la empresa social estaría ofreciendo un ejemplo vivo de posibilidades empresariales que contrastan con los modelos dominantes y que se alinean con la imperiosa necesidad de la transición a la sostenibilidad.

De ahí que este trabajo se haya dirigido a defender la oportunidad que la idea de empresa social representa para experimentar un camino eficaz a seguir ante los retos de la insostenibilidad del modelo económico dominante y ante la necesidad de revisar críticamente las bases del funcionamiento de las empresas convencionales. Oportunidad que se presenta desde tres perspectivas: 1) el cuestionamiento de principios básicos que rigen el paradigma económico vigente es consustancial al emprendimiento social; 2) representa un ejemplo de innovación en el nivel del nicho tecnológico dentro del campo de las transiciones a la sostenibilidad; y 3) la misión social que emerge desde una mente compasiva hace del emprendimiento social un espacio favorable para que puedan prosperar unas bases más democráticas en el gobierno de la empresa y un ejercicio de responsabilidad más auténtico. 
Obsérvese que mencionamos el emprendimiento social no como la vía definitiva para abordar los retos de la crisis ecosocial, sino como un camino más, y altamente condicionado por sus contextos. Su potencia para dejar obsoleto un modelo de empresa guiado por un fin primordialmente económico, generador de múltiples externalidades y sustentado por la visión egoísta de un ser humano centrado en sus posesiones, resulta limitada. El problema de la insostenibilidad global es complejo y vinculado a estructuras sociales y mentales fuertemente arraigadas. Por ello, abordarlo requiere una mirada también compleja, sistémica e integradora, desde la cual la evolución del capitalismo se puede definir como un proceso de influencia recíproca de diversas esferas (Harvey, 2010): concepciones mentales del mundo; reproducción de la vida cotidiana; tecnologías y formas organizacionales; comportamiento humano y relaciones sociales; procesos productivos y laborales; mecanismos institucionales; y relaciones con la naturaleza.

Contextualizar el papel de la empresa social como parte de esa perspectiva coevolucionaria pasará por cambios esenciales en infraestructuras físicas, económicas e institucionales que permitan la creación de espacios para la experimentación, así como recursos, rutinas o mecanismos de presión que se alineen con las nuevas creencias que fundamentan esta nueva configuración empresarial, favoreciendo así la capacidad de acción de los pioneros. En definitiva, la comprensión del emprendimiento social desde tal mirada holística se presenta como condición necesaria para que las empresas sociales puedan enriquecer su capacidad de ampliar los caminos de la transición a una vida buena dentro de los límites planetarios.

\section{BIBLIOGRAFÍA.}

AGLIETTA, M. y REBÉRIOUX, A. (2005), Corporate Governance Adrift. A Critique of Shareholder Value, Edward Elgar, Cheltenham.

ASHOKA (2013), La estrategia de Ashoka para evaluar el impacto. Disponible en http://ashoka.es/sites/spain.ashoka.org/files/EstudioImpacto_2013.pdf (acceso: 12/12/2013).

ASHOKA (2012), Changemaking 101: A student guide to social entrepreneurship, Ashoka y Algernon Sydney Sullivan Foundation, Arlington.

BARTILSON, S. (2012), Social franchising. Obtaining higher returns from investments for jobs in social enterprises, European Social Franchising Network, Northumberland.

BENDELL, J. (2004), Barricades and Boardrooms. A Contemporary History of the Corporate Accountability Movement, UNRISD, Ginebra.

BLOWFIELD, M. y FRYNAS, J.G. (2005), "Setting new agendas: critical perspectives on Corporate Social Responsibility in the developing world", International Affairs, vol.81, núm.3, pp. 499-531. 
BLOWFIELD, M. y MURRAY, A. (2011), Corporate responsibility, 2ª ed, Oxford Universtiy Press, Oxford.

BORNSTEIN, D. y DAVIS, S. (2010), Social Entrepreneurship: What Everyone Needs to Know, Oxford University Press, Nueva York.

CASE (2008), Developing the Field of Social Entrepreneurship. Disponible en http://www.caseatduke.org/documents/CASE_Field-Building_Report_ June08.pdf (acceso: 8/12/2013).

CAVAGNARO, E. y CURIEL, G. (2012), The three levels of sustainability, Greenleaf Publishing, Sheffield.

COMISIÓN EUROPEA (2013), Social economy and social entrepreneurship. Social Europe guide (vol. 4), Dirección General de Empleo, Asuntos Sociales e Inclusión.

CORPORATE WATCH (2006). What's wrong with corporate social responsibility?, Corporate Watch, Oxford.

DEES, J.G. (2007), "Taking social entrepreneurship seriously", Society, vol. 44, núm. 3, pp. 24-31.

DEES, J.G.(2001), The Meaning of Social Entrepreneurship. Disponible en http:// www.caseatduke.org/documents/dees_sedef.pdf (acceso: 3/12/2013).

DESCARTES, R. (2005[1637]), Discurso del método, Edaf, Madrid.

DRAYTON, B. (2005), "Everyone a changemaker", Peer Review, vol. 7, núm. 3 , pp. 8-11.

DUCCI, G. y otros (2002), "The social enterprise in Europe. The state of the art", International Journal of Mental Health, vol. 31, núm.2, pp. 76-91.

EHRENFELD, J.R. y HOFFMAN, A.J. (2013), Flourishing. A frank conversation about sustainability, Greenleaf Publishing, Sheffield.

ENGELMAN, R. (2013), "Beyond sustainababble", en The Worldwatch Institute, State of the World 2013. Is sustainability still possible?, Island Press, Washington, pp. 3-16.

EWING B. y otros (2010), Calculation Methodology for the National Footprint Accounts, 2010 Edition, Global Footprint Network, Oakland.

FROMM, E. (1980), ¿Tener o ser?, Fondo de Cultura Económica, Madrid.

GEELS, F.W. y SCHOT, J. (2010), "The dynamics of socio-technical transitions: a socio-technical perspective in transitions to sustainable development", en Grin, J. y otros (eds.), Transitions to sustainable development. New directions in the study of long term transformative change, Routledge, Londres, pp. 9-101.

GEELS, F.W. (2004), Understanding system innovations: a critical literature review and a conceptual synthesis, en Elzen, B. y otros (eds), System innovation and the transition to sustainability. Theory, evidence and policy, Edward Elgar, Northampton, pp. 19-47.

GHOSHAL, S. (2005), "Bad management theories are destroying good management practices", Academy of Management Learning $\mathcal{E}$ Education, vol. 4, núm. 1, pp. 75-91. 
HANDY, C. (2002), "What's a business for?", Harvard Business Review, vol. 80, núm. 12, pp. 49-55.

HARVEY, D. (2010), The enigma of capital and the crises of capitalism, Profile Books, Londres.

HIRSCH HADORN, G. y otros (eds.) (2008), Handbook of transdisciplinary research. Dordrecht: Springer.

HOLDEN, M. (2008), "The tough minded and the tender minded: A pragmatic turn for sustainable development planning and policy", Planning Theory E̊ Practice, vol. 9, núm. 4, pp. 475-496.

JAMES, W. (2008), Pragmatism: A new name for some old ways of thinking. A series of lectures, Arc Manor, Rockville MD.

JENKINS, W. (2011), "Environmental pragmatism, adaptive management, and cultural reform", Ethics $\mathcal{E}$ The Environment, vol.16, núm. 1, pp. 51-73.

LEVIN, M. y GREENWOOD, D. (2001), Pragmatic action research and the struggle to transform universities into learning communities, en Reason, P. y Bradbury, H. (eds.), Handbook of action research: Participative Inquiry and Practice, Sage Publications, Londres, pp. 103-113.

LIGTH, P.C. (2008), The Search for Social Entrepreneurship, The Brookings Institution, Washington DC.

MATURANA, H. R. y VARELA, F. J. (1987), The tree of knowledge: The biological roots of human understanding, New Science Library, Boston.

MERINO, A. (coord.) (2012), Guía del emprendedor social. Inspiraciones para la creación de empresas al servicio de la sociedad, Universidad Pontificia Comillas, Madrid.

MILL, J.S. (1848), Principles of political economy. With some of their applications to social philosophy (vol. 2), Charles C. Little \& James Brown, Boston.

MILLENNIUM ECOSYSTEM ASSESSMENT (2005), Ecosystems and human well-being, The Millennium Ecosystem Assessment Series, Island Press, Washington DC.

MILLER, C. (2013), Trust, Demand and Last Mile Distribution: The Role of Headteachers in Building Africa's Market for Portable Solar Lights. Disponible en http://www.solar-aid.org/assets/Uploads/Publications/Small-PV-Conference-Paper.pdf (acceso: 9/12/2013).

MINTEER, B.A. (2008), "Pragmatism, piety, and environmental ethics, Worldview: Global Religions, Culture and Ecology, núm. 12, pp. 179-196.

MORIN, E. (1993), "El desafío de la globalidad", Archipiélago: Cuadernos de crítica de la cultura, núm. 16, pp. 66-72.

NÆSS, A. (1995). Self-realization: An ecological approach to being in the world, en Sessions, G. (ed.), Deep ecology for the 21 st century, Shambhala, Boston, pp. 225-239. 
NAREDO, J.M. (2006), Raíces económicas del deterioro ecológico y social. Más allá de los dogmas, Siglo XXI, Madrid.

NAREDO, J.M. (2003), La economía en evolución. Historia y perspectivas de las categorías básicas del pensamiento económico, $3^{\text {a }}$ ed, Siglo XXI, Madrid.

NEF (2005), Ethical Business. How a new breed of business is changing the world, New Economics Foundation, Londres.

NEF (2006), Growth isn't working. The unbalanced distribution of benefits and costs from economic growth. Londres, New Economics Foundation, Londres.

NORGAARD, R.B. (1994), Development betrayed. The end of progress and a coevolutionary revisioning of the future, Routledge, Londres.

PARKIN, S.(2010). The positive deviant: Sustainability leadership in a perverse world, Earthscan, Nueva York.

RECIO, A. (2010), "Capitalismo español: La inevitable crisis de un modelo insostenible”, Revista de Economía Crítica, núm. 9, pp. 198-222.

RIECHMANN, J. (2009), La habitación de Pascal. Ensayos para fundamentar éticas de suficiencia y políticas de autocontención, Los Libros de la Catarata, Madrid.

RIECHMANN, J. y otros (2012), Qué hacemos frente a la crisis ecológica, Akal, Madrid.

ROCKSTRÖM, J. y otros (2009), "Planetary boundaries: exploring the safe operating space for humanity", Ecology and Society, vol. 14, núm. 2, art. 32.

ROTMANS, J. y otros (2001), Transitions and transition management. The case for a low emission energy supply, ICIS Working Paper: I01-E001, ICIS, Maastricht.

SELUSI (2010), Informe personalizado de resultados (España). Disponible en http://www.selusi.eu/uploads/images/101217_Selusi_Report_ES.pdf (acceso: 14/12/2013).

SENGE, P. y otros (2009), "Revolutionary Thinking", Leadership Excellence, vol. 26, núm.12, pp. 10-12.

SHORT, J.C. y otros (2009), "Research in social entrepreneurship: past contributions and future opportunities", Strategic Entrepreneurship Journal, nm .3, pp. 161-194.

SWANSON, L.A. (2010), "The social entrepreneurship zone", Journal of Nonprofit $\mathcal{E}$ Public Sector Marketing, núm. 22, pp. 71-88.

UTTING, P. (2005), Rethinking business regulation. From self-regulation to social control, UNRISD, Ginebra.

VALDALISO, J. M. y LÓPEZ, S. (2000), Historia económica de la empresa, Crítica, Barcelona.

YUNUS, M. (2011), Las empresas sociales. Una nueva dimensión del capitalismo para atender las necesidades más acuciantes de la humanidad, Paidós, Barcelona.

YUNUS, M. (2008). Un mundo sin pobreza. Las empresas sociales y el futuro del capitalismo, Paidós, Barcelona. 